\title{
Concept analysis of spiritual nursing care: a hybrid model
}

\author{
Mozhgan Rahnama', Ahmad Nasiri², Hossien Shahdadi ${ }^{3}$ Mozhgan Jahantigh ${ }^{4}$
}

'Department of Nursing, Zabol University of Medical Sciences, Zabol, Iran

${ }^{2}$ Social Determinants of Health Research Centre, Birjand University of Medical Sciences, Birjand, Iran

${ }^{3} \mathrm{PhD}$ Student of Nursing, Birjand University of Medical Sciences, Birjand, Iran

${ }^{4}$ Community Nursing Research Center, Zahedan University of Medical Sciences, Zahedan, Iran

\section{Abstract}

Introduction: Spiritual care is an important concept in nursing, and there have been different definitions for it; however, in order to address the issue of care, there is a need for a common understanding of this concept. Therefore, the aim of the present study was to analyse the concept of spiritual care in nursing.

Material and methods: the concept of spiritual nursing care was analysed using a hybrid model, which consists of three phases of theoretical, field work, and final analysis. In the theoretical phase, related articles were reviewed in valid databases from 2000 to 2019 . In the field-work phase, in-depth and semi-structured interviews were conducted with 12 participants using a purposive sampling method. Finally, the final analysis was performed by combining the two previous phases.

Results: The results of the final analysis showed that spiritual care is a kind of planned and responsible care through understanding emotions, and psychological and religious needs, which is formed through a trust and empathy-based relationship to solve the patient's problems and focuses on maintaining patients' dignity and respect and establishes a kind of balance between different physical, mental, and social dimensions of the patient.

Conclusions: The results of the current study help to clarify the concept of spiritual care, and in turn provide us with a holistic view of the concept and better understanding of its use in research and practice; however, it is recommended that further studies are carried out in other medical centres in the country to clarify all aspects of this concept.

Key words: spiritual care, nursing, concept analysis, hybrid model.

Address for correspondence:

Dr. Ahmad Nasiri, Social Determinants of Health Research Centre, Birjand University of Medical Sciences, Birjand, Iran, e-mail: anasiri870@yahoo.com

\section{INTRODUCTION}

To maintain health, it is essential to maintain a healthy balance between our mind, body, and soul. In addition to the physical, psychological, and social dimensions, the spiritual dimension is also of interest from the perspective of holistic care [1]. For this reason, available health resources are increasingly highlighting spirituality and its importance in health and disease [2] and encouraging nurses to care for the individual wholeness in four areas: physical, mental, social, and spiritual. However, in practice, the spiritual area is neglected compared to other areas [3], while in order to meet the needs of patients in a holistic manner, spiritual nursing care should be considered as important as other areas of care [4]. The expert panel for the Institute of Medicine, the National Organisation for Nursing Homes, the Palliative Care Organisation (PCO), the Joint Commission for Health Validation [5], and the International Association of Nurses [6] have also advocated for attention to patients' spiritual needs as a standard of practice. Spirituality and spiritual care are regarded as well-known aspects of patient care [7]. In other words, expert care of critically ill patients not only involves paying attention to their physical and mental suffering, but also their spiritual pain [8]. Therefore, nurses and health professionals should play an active role in meeting the spiritual needs of patients along with family members and clergymen [9]. However, studies show that despite the need to provide spiritual care and its reflection in national and international codes of ethics [2], it seems that a large number of nurses have not understood the importance and value of spirituality in care [10]. Adib-Hajbaghery writes that only $27 \%$ of nurses 
regularly and almost half of them rarely provide spiritual care to patients, and this care is ignored in most cases [11]. Paal referred to the increasing need to training spiritual care and gaining competence in this field [12]. Deal also writes that although nurses tend to provide spiritual care, they are not well prepared to do so, and there are also barriers such as unclear roles in this regard [13]. In fact, there are problems with the concept of spiritual nursing care both in theory and in practice [14]. Studies seeking the cause of these problems showed that problems in explicit definition of the concept of spiritual nursing care, nurses' lack of understanding of this concept [15], lack of understanding of the importance and value of spirituality in care [10], lack of knowledge and skills in providing spiritual care [7], lack of specialised training in this field, especially empirical training, and lack of spirituality content in nursing reference books [16] have been cited as reasons for neglecting spiritual care, which indicates the need to clarify and eliminate the ambiguity regarding the concept of spiritual nursing care. The ability of nurses to provide spiritual care and meet the spiritual needs of patients depends on the correct understanding of this concept, and an important barrier to providing spiritual nursing care is the absence of a clear spiritual nursing care concept [17]. However, the number and quality of nursing studies related to spirituality and spiritual care are significantly lower than those focusing on aspects of nursing care in the physical, psychological, and social fields [18]. However, studies have shown that new studies are needed to identify the spiritual care components [10], and providing a definition of spiritual nursing care [17] and providing spiritual care [10] is a nursing challenge. Thus, considering the importance of this issue, there is a need to further develop the concept of spiritual nursing care.

Many approaches to concept development have been used by researchers, including the concept analysis approach, which, as one of the familiar strategies in concept development, investigates the basic elements of a concept in order to determine its nature and function. Concept analysis also leads to the sorting, classification, and adaptation of phenomena, achieving a common understanding of phenomena, preventing personal biases, and preventing conflicts, clarifying many hidden issues, and ultimately strengthening the nursing field. A hybrid model is one of the concept analysis methods that is based on literature review as well as the lived experiences of the participants, and it draws a clear picture of the concepts based on the specific context and situation. Also, because spiritual nursing care depends on the situation and context in which nurses work as well as the organisational culture governing the health institution, nurses' beliefs [19]; therefore, the aim of the present study was to analyse concept of the spiritual nursing care based on a hybrid model to carefully investigate the nursing care concept based on the literature and the experiences of nurses in the real care environment as well as the specific culture of Iran and finally provide a more holistic definition of this concept.

\section{MATERIAL AND METHODS}

In this study, a hybrid model has been used to analyse the concept of spiritual nursing care. This method is used in nursing to eliminate abstraction and ambiguity, and it draws a clear picture of concepts based on the specific context and situation and is superior to other methods of concept analysis in this regard. This model consists of three stages of theoretical, field work, and analytical phases.

In the theoretical phase, much emphasis is placed on the intrinsic nature of the concept, and the operational definition for the field-work phase is determined at the end. The field-work phase emphasises the experimental component of the process, and the qualitative data collection method is used in order to more fully analyse the concept. The comprehensive review of articles that started in the theoretical phase continues in this stage, and this review is maintained as a basis for comparing the data collected in the field. The third phase helps to summarise the analysis and provide possible suggestions to redefine the concept using an analytical approach [19].

\section{Theoretical phase}

At this phase, to examine the spiritual nursing care concept, all valid databases such as PubMed, CINAHAL, Science direct, Google Scholar, SID, Magiran, ProQuest, and Medline were searched using the keywords Concept analysis, Spiritual care, Nursing, and all possible combinations of these words, from 2000 to 2019. Selection criteria included original and review research articles written in English or Persian, the content of which had a definition and explanation of spiritual nursing care, as well as access to the full text of the article. In the initial review, 532 articles were found, of which 250 were full-text articles and 282 were available as summaries. After carefully reviewing the articles based on the purpose of the study and removing duplicate and irrelevant articles, 16 full-text articles were finally used.

\section{Field-work phase}

In this study, purposive sampling was used to select the nurses working in the hospital and nursing professors of the faculty who had sufficient information and experience regarding the phenomenon 
in question, and face-to-face interviews were then carried out (it should be noted that the selection of participants continued until reaching data saturation where no new content was added to the content of the previous category). Semi-structured and in-depth interviews were used for this purpose. Interviews lasted 45 to 60 minutes according to the willingness and mental and physical conditions of the participants in a private room in Ali ibn Abi Talib Hospital of Zahedan. Also, face-to-face interviews were carried out individually with participants outside of their working times, and they were asked to answer the researcher's questions either by telephone or, if possible, by re-attending the interview location. All participants were asked three main questions: "What is your definition of spiritual nursing care?", "What are the aspects of spiritual nursing care?", and "What comes to mind when you hear about spiritual nursing care?" Based on the answers of the participants, more questions were asked to discover the different dimensions of this concept: "Please give an example in this regard?", "What do you mean by that?", "Can you explain further?" At the same time, in order to observe maximum variations, efforts were made to select participants from different wards, with different years of work experience, and of both sexes.

In this research, data analysis and data collection were carried out simultaneously using a conventional content analysis method and based on the five steps proposed by Graneheim and Lundman: 1. Transcribing the whole interview immediately after each interview (Transcribing); 2 . Reading the whole interview text to gain a general understanding of its content and to determine the meaning units and primary codes; 3 . Abstracting the meaning units and primary codes; 4 . Sorting similar primary codes in more comprehensive classes; and 5. Determining the hidden data content.

Lincoln and Guba's (1985) criteria were used to ensure the trustworthiness and rigor of the present study. In this study, in order to ensure the credibility of findings, the researcher met with the participants several times before the interview so that she/he could gain the participants' trust and create a suitable relationship and atmosphere for in-depth interviews. Member checking was also used to validate the trustworthiness of the research findings.

In order to ensure confirmability of the findings, at the time of data collection, the researcher tried to collect the information carefully and thoughtfully, avoid any bias, and use people to participate in the research who could provide important information. Also, wherever the researcher felt that he or she did not have sufficient and appropriate information about some of the patient's statements, he/she would refer to those statements again at the appro- priate time during the interview so that the patient could give more information to the researcher.

For the transferability of the findings, attempts were made to use patients with different demographic characteristics and different experiences and take into account all aspects of behaviours, events, and lived experiences.

With regard to the dependability of the findings, part of the interview text along with the relevant codes and the emerging classes were sent to several observers to review the analysis process and comment on their accuracy. Also, all activities and decisions, including data collection and analysis, were recorded separately and included in the article text to follow in the research for others, if necessary.

In this study, attempts were made to fully explain the research purpose and methodology to the participants and assure them that their identities would not be revealed during the research and dissemination of findings. Also, to maintain the anonymity of individuals, one number was assigned to each of them, and people were quoted based on these numbers, and they were assured that they could withdraw from the study if they did not want to continue cooperating at any stage of the research. In addition, written informed consent was obtained from the participants.

\section{Final stage}

In this stage, the definitions and concepts obtained from the previous two stages were merged, and finally a comprehensive and complete definition, which included all the characteristics presented in the previous stages, was presented.

\section{RESULTS}

\section{Results of the theoretical phase}

The results of the literature review showed that spiritual nursing care is a complex concept that is difficult to define precisely (Table 1). The Oxford Dictionary defines the word "spirituality" as a fact or condition of the soul or composed of immaterial essence, the quality or state of being spiritual, and "spiritual" as belonging to, or in relation to, the soul or high moral qualities, or about or in relation to sanctities [20].

Moosavi defines spiritual care as "including the need for meaning, self-expression, sanctities, and religious practices when faced with illness, grief, and sorrow" [21]. Hummel and Galek also believe that "Religious interventions are a major part of nurses' spiritual care and include interventions such as praying with patients, talking to patients about God, discussing religion with patients, and provid- 
Table 1. Summary of literature review

\section{Author Name}

Govier (2000)

\section{Conceptz}

Spiritual care in the form of "reasoning", "thinking", which refers to the search for meaning and purpose in life experiences, "restoration", which refers to the ability of the spiritual dimensions to influence physical aspects, "religion", which is a way to show spirituality through a framework of values and beliefs, and "communications", which refer to the desire for a relationship with oneself, others, and a superior being (God) that may be manifested through love, trust, hope, and being of service to others.

Mc Sherry (2002) Spiritual care includes maintaining the patient's privacy and dignity, listening intelligently to the patient, enabling the patient to perform his or her religious activities in the hospital, and helping him or her to find meaning and purpose in the disease.

Sawatzky (2005) Spiritual care is an integrated intuitive, interpersonal, and humanitarian manifestation that depends on nurses' awareness of the transcendental dimensions of life and reflects the reality of patients, and more specific behavioural interventions should reflect patients' spiritual beliefs. In other words, spiritual nursing care is a self-manifestation. "Spiritual manifestations such as love, hope, and intimacy constitute the most basic and general approach to spiritual care and can be integrated into all aspects of nursing care", he writes.

Hanson (2008) He referred to helping to communicate with friends, gain peace by loving others, and helping to communicate with God by helping to participate in religious / spiritual services, helping with religious / spiritual activities, helping to pray and say prayers as essential activities during spiritual care.

Daaleman He introduced the physical and mental presence of the caregiver and his / her awareness of the patient's life (2008) stages and experiences related to the patient's disease through a fluid two-way communication between the patient, family members, and caregivers that leads to a care focusing on maintaining patient dignity and respect as spiritual care.

Hummel (2008) To define spiritual care, they referred to 10 categories of spiritual interventions: 1. Religious interventions; 2. Spiritual interventions; 3 . Counselling; 4. Emotional support; 5 . Defence and support; 6 . Presence; 7 . Respect; 8. Communication; 9. Sub-therapies (art therapy, touch therapy); 10. Other care with religious interventions accounting for the largest category, which includes interventions such as praying with patients, talking to patients about God, religious discussion with patients, providing religious support, providing religious books and other related supplies, providing religious music, encouraging religious activities, referring patients to religious resources, praying for patients, identifying how patients relate to God, and identifying patients' images of God. Spiritual interventions also include facilitating meaning and purpose, enabling the individual to strengthen existence, supporting spiritual search, suggesting spiritual resources, providing spiritual guidance, promoting spiritual communication (companionship and empathy), and providing inspirational books.

Conway (2010) Spiritual care involves intimacy, presence, listening, and guiding people toward real hope, and may not involve the discussion of religion at all.

Deal (2010) Spiritual care includes the following: 1. Patient-centred care because it is designed based on the patient's needs; 2. Simple care because it includes simple interventions such as talking to the patients, listening to them, and expressing positive expressions; 3 . It is a care welcomed by patients; 4 . It is care with various providers, because anyone who is in contact with the patient can play a role in creating a relationship that leads to relaxation.

Mahmo- Spiritual care focuses on respect for the patient, friendly and empathetic interactions, participation in religious odishan (2010) services, and increasing the inner strength of patients and nurses.

Monareng He defined the concept of spiritual care using the concepts of "caring presence", which refers to (2012) the availability, listening, touching, and providing spiritual support; "search for meaning and purpose", which focuses on the and finding the meaning of human existence; "metaphysics", which is the process of going beyond existing limitations and finding a new perspective on self and existence; "harmonious communication", which is shown through proper communication with God, self, and others; and "spiritual dialogue", which includes the part of nursing care that includes talking to patients who experience spiritual distress.

Monareng Spiritual nursing care includes activities that facilitate the establishment of a kind of balance between different

(2013) physical, mental, social, and spiritual dimensions in a person and lead to a feeling of perfection and health.

Ramezani

(2014) They defined spiritual nursing care in the caring presence, providing meaning-based therapeutic interventions, and creating a spiritual environment.

Adib-

(2014)

Spiritual care is defined as activities and methods for providing care that improve the quality of life, spiritual health, and spiritual performance and positively affect the individual's response to stress, balance between physical, mental, social, and spiritual aspects, sense of wholeness, and excellence and improvement in his/her relationships with others.

Chandramohan Spiritual care includes activities including the following: 1. Establishing a trust- based relationship between (2016) the nurse and the patient; 2. Providing a spiritual supportive environment; 3 . Sensitivity to the spiritual and cultural beliefs of the patient and their families; 4. Recognising the importance of presence or therapeutic use of self; 5 . Demonstration (presentation) of care through practical nursing activities; 6 . Incorporation of spirituality into the care program

Moosavi Spiritual care includes the need for meaning, self-worth, faith, prayer, sanities, and religious practices

(2019) in the face of trauma, disease, grief, and sorrow.

Burkhart Spiritual care is a planned process to help others improve their own spirituality and especially to deal (2019) with stressful situations. 
ing religious support, providing religious books and other related supplies, providing religious music, encouraging religious activities, referring patients to religious resources, praying for patients, identifying how patients relate to God, identifying patients' image of God" [22]. McSherry, on the other hand, states that spiritual care "enables people to perform their religious activities in the hospital" [23]. Religion can be regarded as a way to show spirituality through a framework of supreme values [24]. However, spiritual care is not limited to activities that emphasise religion $[13,25]$ and includes counselling, defence and support, secondary therapies such as art therapy, touch therapy, routine care [22], establishing a trustbased relationship, being sensitive to the patient's spiritual and cultural beliefs, and integrating spirituality into the care plan [26]. In fact, it is a kind of help to gain a sense of calm, find a sense of taking control of our life, cope with diseases, eliminate fear of death and dying, alleviate worries about suffering, gain hope, recognise the importance / value of life, understand the meaning of disease, and gain insight to the death experience [5]. It is also a kind of physical and mental presence and thought-based planned activity to provide care that goes beyond medical treatment, during which caregivers are aware of their patient's life stages and personal experiences related to a serious illness. In fact, it is a reciprocal activity between patients, family members, and their caregivers that provides a kind of care maintaining patient dignity and respect [27]. Spiritual care reinforces aspects such as "meaning and purpose in life" [28] and refers to the desire for a relationship with oneself, others, and a superior being (God), and may be displayed through "spiritual manifestations" such as love, trust, hope, intimacy, respect for the patient, friendly and empathetic interactions, and being of service to others $[24,29,30]$. Spiritual care consists of other components including a "caring presence" that refers to availability, listening, and touching and includes the values such as intimacy, respect, empathy, hope, and concern, and "spiritual dialogue", which includes that part of nursing care that includes talking to patients who experience spiritual distress $[17,28]$. This type of care promotes the quality of spiritual life, spiritual health and performance, and has a positive effect on a person's response to stress, feeling of wholeness, and excellence, and improving his/her relationships with others [31]. It also establishes a kind of balance between different physical, mental, social, and spiritual dimensions, especially in response to stressful situations [4,32].

As a general conclusion of the literature review, it can be said that spiritual nursing care is a planned caring presence formed in the form of a trust- and empathy-based relationship between the patient and caregivers. It focuses on the dignity, respect, sanctities, and religious practices of patients and help individuals creates balance between different physical, mental, and social dimensions in the face of stressful situations.

\section{Results of the field-work phase}

This stage of the study was performed on 12 people, including nine nurses and three faculty members (two instructors and one assistant professor) with a mean age of $35.58 \pm 8.02$ years. The majority of them were women $(66.7 \%)$, married $(58.4 \%)$, and had a bachelor's degree $(75 \%)$. Findings obtained from this phase were divided into four themes: "Empathy", "Mental support", "Religious meditation", and "Responsibility".

\section{Empathy}

Participants stated that the nurses must be able to have a proper understanding of the patient's feelings in order to perform spiritual nursing care so that they can help patients in times of need through empathy, consolation, and compassion, and thus provide effective care. In this regard, the participants expressed the following:

"Spiritual nursing care means that you understand the patient's feelings, you can empathise with $\mathrm{him} / \mathrm{her}$. If I, as a nurse, have a good understanding and feeling of the patient, I can provide spiritual care, and I do not have to have special conditions to provide this care" (a 25-year-old male nurse).

"When I feel my patient, it means I was able to hold his/her hand and understand her/him, thereby providing spiritual care to him/her. In fact, if I am not compassionate and sympathetic to the patient and do not sympathise with him/her, it means that I could not understand my patient, I could not communicate with him/her; therefore, I could not take care of her/him" (a 43-year-old female instructor).

\section{Mental support}

A number of participants also expressed a belief that in order for spiritual care to take place, the nurses must be able to meet the mental and psychological needs of patients and induce hope and a sense of calm, and improve their mood in the face of illness through establishing mental and caring relationship with patients.

"In my opinion, spiritual care means to pay attention to the psychological needs of our patients. We need to be able to give hope to a patient who has given up hope, and we can do so if we establish an appropriate relationship with the patient so that he or she can talk to us easily, thereby taking care of him 
or her as well as gaining her/his trust" (a 42-year-old female instructor).

"I try to pay attention to the patients' morale and prepare whatever they feel comfortable with, communicate and be kind with them. Sometimes patients have mental and emotional needs; for example, they want one of their loved ones to be with them". I help this take place; I think it is spiritual care" (a 26-year-old male nurse).

\section{Religious meditation}

Some participants stated that the purpose of spiritual care was to pay attention to religious needs, advise to rely on God, and allow the family to help the patient perform his/her religious duties.

"If we pay attention to the patients' religious needs, it means that we help them in their religious duties, allow the family to perform their religious duties, and give hope to them by the grace and mercy of God. "Even while we want to provide routine care, such as when we want to give them medicine, it is better to pay attention to the patient's beliefs and religion" (a 41-year-old female nurse).

"Sometimes it is Azan time and the patient wants to pray, and he/she asks us where is the prayer hall? What direction is the Qibla?" Or, for example, in a certain situation, he/she wants to pray and ask us for a prayer book or the Qur'an. When he/she raises his/her concerns, we should realise that he/she needs our care here" (a 30-year-old male nurse).

\section{Responsibility}

A number of participants also believed that spiritual care includes actions such as feeling responsible for solving patients' problems, selflessness towards patients, paying attention to patients' demands, giving patients the right to live, and being sensitive to their rights.

"In my view, in order to provide spiritual care, the nurse must remember that all patients have the right to live, be sensitive to their rights, and even dedicate him/herself to help the patient" (a 35-year-old female nurse).

"The best thing we can do as nurses is to solve our patients' problems and do their duties, as one of the great people says that we must be sensitive to the human needs" (a 30-year-old male nurse).

Overall, the result of the second stage of research, field work, was a defined as follows: Spiritual nursing care is a kind of responsible care through understanding emotions, and psychological and religious needs, in which the nurse communicates constructively with the patient and his/her caregivers and does his/her best to help patients be calm and solve their problems.

\section{Final analysis}

In the final stage, according to the findings of the first two previous stages, namely the theoretical and the field-work, the final definition of the concept of spiritual nursing care was presented as follows: Spiritual nursing care is a planned and responsible care through understanding emotions, and psychological and religious needs, in which the nurse attempts to interact constructively with the patients and their caregivers and focuses on their dignity, respect, and sanctities to help the patient be calm and solve their problems as well as establish a balance between different physical, mental, and social dimensions under stressful situations.

\section{DISCUSSION}

The present study was conducted to clarify the concept of spiritual nursing care using a three-stage hybrid model. This concept was clarified through the analysis process and showed that this concept includes a range of characteristics that were identified in two stages of theoretical and field-work phases. The results showed that the concept of spiritual nursing care can be described from the perspective of the nurses under study not only in religious meditation but also as empathy with the patient, emotional support, and a sense of responsibility for the patient's problems. Similarly, Lotfi Kashani et al. also write that spirituality can be proposed in two dimensions: The first dimension is religious spirituality, in which the individual's concept of sacred existence or ultimate reality is expressed in a religious style; and the second dimension, existential spirituality, it one in which specific psychological experiences are considered, which are in fact unrelated to sacred or ultimate existence [33]. Zamanzadeh et al. carried out a qualitative study with the aim of "explaining patients and nurses' perceptions of the concept of spirituality in cancer care". Although one of the themes extracted from the interviews was "religion-based strategies", and accordingly, providing an opportunity to correct beliefs and closeness to God was identified as an aspect of spiritual care, they went on to say that nurses, in addition to using strategies based on religious beliefs to strengthen the spiritual dimension, were a source of energy, happiness, hope, and power for patients by being empathic, kind, and cheerful [34].

The nurses in the Chandramohan et al. study, titled "Application of spirituality and the spiritual care of nurses' activities in South Africa", also believed that providing the opportunity to meet with a religious expert, respecting the patient's privacy, religion, and beliefs, kindness, and spending time with 
the patients, supporting them, listening to them, and giving the patient the opportunity to express their fears and anxieties, as well as enabling them to find meaning and purpose in the disease are examples of providing spiritual care [26]. In a study titled "Nurses' experiences of providing spiritual care", Deal stated that nurses can support patients by providing spiritual interventions such as devoting time to them, listening to them, and praying for them [13]. In the study of Bani-Molham et al. titled "Nurses' understanding of spirituality and spiritual care", nurses also believed that appropriate spiritual care must be based on respecting the patients' beliefs and paying attention to their needs, feelings, and concerns [35]. Clearly, from the point of view of the nurses of these studies, like the nurses of the present study, paying attention to religious needs as well as showing empathy, kindness, giving hope, giving energy, spending time with the patients, listening to them, feeling responsible for their problems, and giving them opportunities to express their feelings are among examples of spiritual nursing care. In this regard, Hosseini writes that spirituality is often confused with religion because it is difficult to distinguish between them. We should be aware that these are two complementary concepts, and religion can be a basis for spiritual manifestations [36].

According to the findings of the present study, spiritual care was defined as a type of planned caring presence that includes physical, mental presence and paves the way for the provision of a care focused on maintaining the patients' dignity and respect and thus the acquisition of meaning and purpose in their lives. Consistent with the above finding, in a study titled "Analysis of the concept of spiritual nursing care", Ramezani et al. also mentioned caring presence and patient-centredness as characteristics of spiritual care and suggested that these interventions should be meaning oriented [28]. Similarly, in his research titled "Analysis of the concept of spiritual nursing care", Monareng referred to empathy, intimacy, respect, hope, and addressing patients' concerns along with the caring presence as values and characteristics of spiritual nursing care [17].

The aim of the present study was to gain a clear understanding of the concept of spiritual nursing care. Analysis of the concept of spiritual nursing care showed that this concept is multidimensional and complex and includes a set of behaviours and characteristics. One of these characteristics is that it is a planned and responsible care and is achieved through understanding the feelings and spiritual, psychological, and religious needs of patients as well as building a constructive interaction between the nurse and the patients and their caregivers and focusing on maintaining their dignity, respect, and sanctities. The nurse also does her/his best to help patients be calm, solve their problems, and establish balance between the various physical, psychological, and social dimensions in stressful situations.

The concept of spiritual nursing care can give policymakers, staff, service providers, and nursing instructors a broad insight into the need to pay attention to spiritual care and its application in the field of educational and clinical contexts. It can also be used as a guide for instructors, clinical nurses, and managers to facilitate the process of providing spiritual care. In other words, we should clarify, to a large extent, the dimensions and characteristics of this concept in nursing, thereby evaluating, researching, and expanding the body of knowledge in nursing. In the meantime, providing nursing services, according to the spiritual dimension, and holistically, will induce satisfaction in patients, thereby evaluating, researching, and expanding the body of knowledge in nursing.

The findings of the present study identified the characteristics of the concept of spiritual care so that many misconceptions and misapplications of this concept can be corrected. However, since the concept analysis is itself an endless process, further studies are needed to clarify the details of this concept, and we hope that the findings of the present study will be the beginning of further studies on spiritual nursing care. One of the limitations of the present study is the selection of nurses and nursing instructors from one religion and culture, and considering the role of religious and cultural activities in spiritual care, it is suggested that the opinions and experiences of nurses from other cultures and religions be investigated in future studies. Moreover, it is suggested that the opinions and experiences of nurses be evaluated regarding spiritual care, as well as those of other caregivers and patients.

\section{ACKNOWLEDGMENTS}

The authors express their gratitude to all the faculty members and nurses of Zahedan University of Medical Sciences for sharing their valuable experience by participating in the present study.

The authors declare no conflict of interest.

\section{REFERENCES}

1. Boero ME, Caviglia ML, Monteverdi R, et al. Spirituality of health workers: a descriptive study. Int J Nurs Stud 2005; 42: 915-921.

2. Rahnama M, Fallahi Khoshknab M, Seyed Bagher Maddah S, et al. Cancer patients' perception of spiritual care. Iran J Med Ethics Hist Med 2012; 5: 64-80. 
3. Rassouli M, Zamanzadeh V, Ghahramanian A, et al. Experiences of patients with cancer and their nurses on the conditions of spiritual care and spiritual interventions in oncology units. Iran J Nur Midwifery Res 2015; 20: 25-33.

4. Monareng LV. An exploration of how spiritual nursing care is applied in clinical nursing practice. Health SA Gesundheid 2013; 18: 1-8.

5. Hanson LC, Dobbs D, Usher BM, et al. Providers and type of spiritual care during serious illness. J Palliat Med 2008; 11: 907-914.

6. Baldacchino DR. Teaching on the spiritual dimension in care to undergraduate nursing students: the content and teaching method. Nurse Educ Today 2008; 28: 550-562.

7. Wong KF, Yau SY. Nurses' experience in spirituality and spiritual care in Hong Kong. Appl Nurs Res 2010; 23: 242-244.

8. Sheehan MN. Spirituality and the care of people with lifethreatening illness. Techn Regional Pain Management 2005; 9: 109-113.

9. Baldacchino D. Spiritual care education of health care professionals. Religions 2015; 6: 594-613.

10. Nacimento LC, Oliveria FCS, Moreno MF, et al. Spiritual care: an essential component of the nurse practice in pediatric oncology. Acta Paul Enferm 2010; 23: 437- 440.

11. Adib-Hajbaghery M, Saeadnejad Z. Barriers to provide patients admitted to hospitals in Kashan with spiritual care: nurses' viewpoint. Med Ethics J 2016; 10: 49-59.

12. Paal P, Roser T, Frick E. Developments in spiritual care education in German-Speaking countries. BMC Med Educ 2014; 14: 112 .

13. Deal B. A pilot study of nurses' experience of giving spiritual care. Qualit Rep 2010; 15: 852-863.

14. Stirling I. The provision of spiritual care in a hospice: moving towards a multi-disciplinary perspective. Scott J Healthcare Chaplinary 2007; 10: 21-27.

15. Mooney B, Timmins F. Spirituality as a universal concept: student experience of learning about spirituality through the medium of art. Nurs Educ Pract 2007; 7: 275-284.

16. Skalla K, McCoy JP. Spiritual assessment of patient with cancer: the moral authority, vocational, aesthetic, social, and transcendent model. Oncol Nurs Forum 2006; 33: 745-751.

17. Monareng LV. Spiritual nursing care: a content analysis. Curationis 2012; 35: 1-9.

18. Moadel A, Morgan C, Fatone A, et al. Seeking meaning and hope: self-reported spiritual and existential needs among an ethically-diverse cancer patient population. Psychooncol 1999; 8: 378-385.

19. Salsali M, Mohammadpour A, Fakhrmovahedi A. The methods of concept development. Tohfe-Boshra Public 2006; P: 83-85.

20. Sessanna L, Finnell D, Jezewski MA. Spirituality in nursing and health-related literature a concept analysis. J Holist Nurs 2007; 25: 252-262.

21. Moosavi S, Rohani C, Borhani F, et al. Consequences of spiritual care for cancer patients and oncology nurses: a qualitative study. Asian Pac J Oncol Nurs 2019; 6: 137-144.

22. Hummel L, Galek K, Murphy KM, et al. Defining spirituality care: an exploratory study. J Health Care Chaplain 2008; 15: 40-51.

23. McSherry W, Draper P, Kendrick D. The construct validity of a rating scale designed to assess spirituality and spiritual care. Int J Nurs Stud 2002; 39: 723-734.

24. Govier I. Spiritual care in nursing a systematic approach. Nurs Stand 2000; 14: 32-36.

25. Conway J. Integrating spiritual care as part of comprehensive cancer treatment. Oncol Nurs Advisor 2010; 24-27.

26. Chandramohan S, Bhagwan R. Utilization of spirituality and spiritual care in nursing practice in public hospitas in KwaZuluNatal/South Africa. Religions 2016; 7: 1-13.
27. Daaleman TP, Usber BM, Williams SW, et al. An exploratory study of spiritual care at the end of life. Ann Fam Med 2008; 6: 406-411.

28. Ramezani M, Ahmadi F, Mohammadi E, et al. Spiritual care in nursing: a concept analysis. Inter Nurs Rev; 61: 211-219.

29. Sawatzky R, Pesut B. Attributes of spiritual care in nursing practice. J Holistic Nurs 2005; 23: 19-33.

30. Mahmoodishan G, Alhani F, Ahmadi F, et al. Iranian nurses' perception of spirituality spiritual care: a qualitative content analysis study. J Med Ethics Hist Med 2010; 3: 1-8.

31. Adib-Hajbaghery M, Zehtababchi S. Assessment of nurses professional competence in spiritual care in Kashan's hospitals in 2014. Sci J Hamadan Nurs Midwifery Faculty 2015; 22: 23-32.

32. Burkhart L, Bretschneider A, Gerc S, et al. Spiritual care in nursing practice in veteran health care. Global Qualitat Nurs Res 2019; 6: 1-9.

33. Lotfi Kashani F, Vaziri SH, Gheisar SH, et al. Effectiveness of spiritual interventions to reduce maternal quality of life in children with cancer. Quarterly J Med Figh 2012; 4: 125-149.

34. Zamanzadeh V, Ghahramanian A, Rassouli M, et.al. Spirituality in cancer care: a qualitative study. J Qualitat Res Health Sci 2013; 4: 366-378.

35. Bani Melhem GH-A, Zeilani R-S, Zaqqout O-A, et al. Nurses' perceptions of spirituality and spiritual care giving: a comparision study among all health care sectors in Jordan. Indian J Palliat Care 2016; 22: 42-49.

36. Hosseini M, Davidson PM, Fallahi Khoshknab M, et al. Spiritual and religious interventions in health care: an integrative review. Iran Rehanilitation J 2013; 11: 87- 93. 